Journal of Computer Science 8 (4): 454-459, 2012

ISSN 1549-3636

(C) 2012 Science Publications

\title{
Multipath Adaptive Packet Dispersion for Voice Applications
}

\author{
${ }^{1}$ Faritha Banu, J. and ${ }^{2}$ V. Ramachandran \\ ${ }^{1}$ Department of CSE, Sathyabama University, Chennai, India \\ ${ }^{2}$ Department of IT, Anna University, Chennai, India
}

\begin{abstract}
Problem statement: The quality of Voice over IP applications is decided by the main factors like network capacity and technologies such as codec type, Packet Loss Concealment (PLC) and Forward Error Correction (FEC). Approach: In MPLS networks multiple Label Switched Paths (LSP) are established between MPLS ingress and egress nodes to enhance the network performance and QoS for subscribers. To enhance the Quality of Service, Multipath adaptive packet dispersion is proposed for voice applications in IP networks. Results: The trigger handler is proposed to check the balanced load in the system. If the network condition becomes unbalanced, the adaptive packet scheduler classifies the flows and routed to the best shortest paths with the help of proposed packet dispersion technique. Conclusion: The VoIP traffic is routed along the most adequate path that has enough resource to meet a given target QoS. Simulation results are reported to show the efficiency of the proposed technique for effective VOIP flows.
\end{abstract}

Key words: MPLS, load balancing, VoIP and QoS, broadband scenario, voice data, voice applications, service providers, multipath packet dispersion technique, voice communication, probe packets, splitting algorithm

\section{INTRODUCTION}

VoIP networks: Conventionally packet switched networks are termed as IP networks having basic characteristics of directing the traffic over the best shortest path, where the application divides the stream into packets at the source and forwards them towards the destination (Huanga et al., 2006). The transport of voice data as packets over IP based networks is termed as voice over IP (VoIP). Hence, VoIP can be obtained on any network that is using IP such as the Internet, intranets and local networks in which digitized voice packets are passed over the IP networks (Fernandez et al., 2007). The VoIP networks (Guillen and Chacon, 2009) guarantee to maintain a reliable voice communication in a broadband scenario further enhancing the best cost-benefit relationship. The service providers and users are accomplished with a Service Level Agreement (SLA) which specifies allowable values of QoS parameters across the domains they control. These parameters to be guaranteed to the users as specified in the SLA.

Motivation and proposed solution: Networks are prone to failures because of unreliable equipments, software bugs or cable cut and other similar reasons. Such faults and failures may affect the operation of label switched paths and cause packet loss. In case of failures, in order to balance the load, packets are moved from the affected LSPs to the non-affected LSPs. This study proposes a new MPLS based adaptive multipath packet dispersion technique for load balancing and to enhance the performance of QoS metrics. Multipath routing based dispersion involves traffic splitting that consists of dividing each flow of packet stream along two or more disjoint paths such that the sum of available bandwidths of these paths satisfies the proposed bandwidth requirement of each type of flow, depending on the type of traffic.

The packet dispersion mechanism, resides on a router that splits the VoIP flows over the MPLS based multiple parallel paths. Since the VoIP traffic is dispersed along multiple parallel paths which satisfy the given QoS, load balancing is achieved in the system, thereby providing fair bandwidth allocations.

Related works: Load balancing can be adaptive or non adaptive. Adaptive load balancing policies use real time system state information based on various metrics like bandwidth, free available memory to take load balancing decisions (Kambourakis et al., 2006). Non adaptive or static load balancing policies do not use real time system state information's. Hanoch (Levy and Zlatokrilov, 2006; Zlatokrilov and Levy, 2004), proposed a model in which packets of a certain session are dispersed over multiple paths, in contrast to the traditional approach. The 
dispersion may be performed by network nodes for various reasons such as for balancing the load or implemented as a mechanism to improve Quality of Service, as will be presented in this study.

To study the effect of packet dispersion on the quality of Voice over IP applications, they have focused on the network performance by calculating the loss rate and loss burstiness on the applications. Packet Loss Rate often called as Noticeable Loss Rate (NLR) metrics is proposed by IETF. It counts losses of "Close" packets and ignores losses of distant packets. They have analyzed NLR metrics for various packet dispersion strategies over memoryless (Bernoulli) loss model or bursty (Gilbert) loss model.

Some other related works have designed with the goal of dynamic load balancing (Kandula et al., 2007) to prevent network from congestion and packet losses. This approach introduces the concept of flowlet (burst of packets) splitting that is based on per packet based splitting but avoids packet reordering. Packet loss is one of the major difficulties faced by VoIP applications. To overcome the packet loss receiver based packet loss concealment strategy (Maheswari and Punithavalli, 2009) is introduced. In which previously arrived segment of voice samples are used to reconstruct the missing packet and it is played out to readjust the packet loss. This mechanism fails when the packet size is large and the loss rate is high. A node based multipath routing and congestion control approach (Paganini and Mallada, 2009) uses "backpressure" scheduling to measure congestion prizes. Based on these congestion measures traffic flows are splitted dynamically and dispersed to multiple paths.

Several traffic splitting algorithm like flow based, packet based, S-Hash splitting and Bin-based splitting were proposed to split the traffic flow into multiple paths. One such packet based splitting approach (Avallone et al., 2007) is used to improve network security and resource utilization in MPLS networks. The idea is based on splitting of traffic flows on a per-packet basis at the ingress of an MPLS cloud and disperses them to multiple paths by adding flow label. Splitting the packets belonging to particular flow over multiple parallel paths may arrive out of order in the egress Label Edge Router (LER). Egress LER receives labeled packets, removes MPLS header and passes them on to the IP layer. Reordering mechanism at the egress LER is essential in this approach. This is achieved by inserting splitting id in the TTL field of the MPLS header along with the sequence number in the label filed at the ingress router (Molnar et al., 2009).

\section{MATERIALS AND METHODS}

Proposed multipath packet dispersion technique: The proposed technique is mainly dealt with flow classification and implements load adapter with effective triggering policies. Deterministic Periodic packet dispersion scheduler resides in a router that routes VoIP packets into multiple QoS guaranteed parallel paths. The entire steps involved in the proposed technique are depicted in the following flowchart Fig. 1.

Flow classification: Traffic flows are classified as VoIP flow and Normal flow by using edge-to-edge probing. As a first step in the flow classification, the link which posses high losses, bandwidth are to be identified. For this the ingress router of each LSP periodically sends a back-to-back (a probe pair) probe packets on all the forward LSP from ingress to egress. Egress router receives these probe packets at different intervals (Gap). Some of the probe packets can be lost due to congestion or link failures. Using the sequence number, the number of lost packets and the gap between them are estimated. By measuring the gap, the available link bandwidth is also estimated. Each probe packet is associated with a label, which defines a flow arrival rate and the id of the router through which the flows are entering into the domain. The egress router sends feedback packets containing the measured packet loss and bandwidth periodically back to the ingress router.

In this scheme, source sends a series of probe packets along a path $\mathrm{P}$ to the destination, with no delay among the transmissions of successive packets.

The loss ratio $\left(\mathrm{L}_{\mathrm{Ri}}\right)$ at each node $\mathrm{n}_{\mathrm{i}}$ along $\mathrm{P}$ at the interval $\mathrm{t}$ seconds can be calculated as Eq. 1:

$\mathrm{L}_{\mathrm{Ri}}{ }^{\mathrm{t}}=\mathrm{PL} / \mathrm{R}$

Where:

$\mathrm{PL}=$ The number of packets lost

$\mathrm{R}=$ The estimated arrival rate of the packet

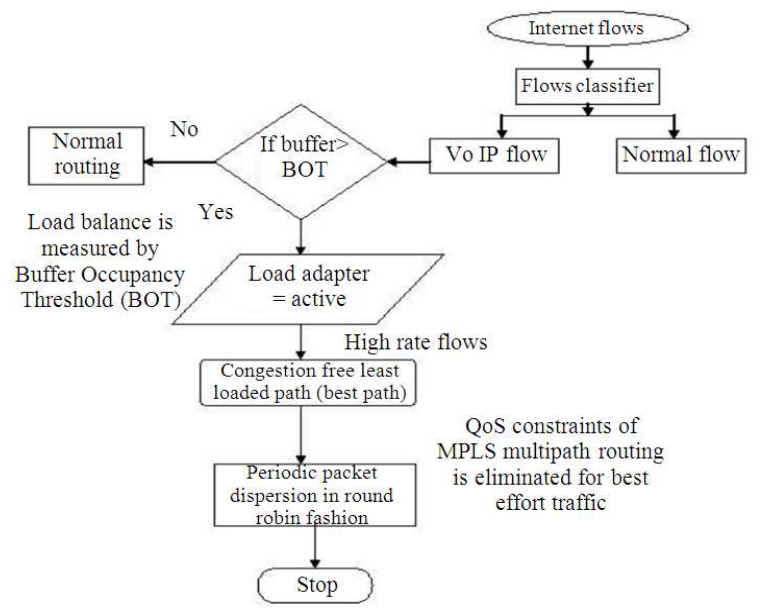

Fig. 1: Flow chart of the Proposed System 
Then the total loss ratio at destination can be calculated as Eq. 2:

$\mathrm{L}_{\mathrm{R}}{ }^{\mathrm{t}}=\sum \mathrm{L}_{\mathrm{Ri}}{ }^{\mathrm{t}}$

Now the actual traffic flows are transmitted for the same sample interval of $t$ seconds and the packet loss rate is measured.

The actual loss ratio $\left(\mathrm{L}_{\mathrm{Ract}}\right)$ at each node along $\mathrm{R}_{1}$ at the interval $\mathrm{t}$ Seconds can be estimated similarly as (Eq. 1).

Then the total actual loss ratio at destination can be calculated as Eq. 3:

$\mathrm{L}_{\text {Ract }}{ }^{\mathrm{t}}=\sum \mathrm{L}_{\text {Racti }}{ }^{\mathrm{t}}$

At egress router, the difference in loss ratios can be then estimated as Eq. 4:

$\mathrm{D}=\mathrm{L}_{\text {Ract }}{ }^{\mathrm{t}}-\mathrm{L}_{\mathrm{R}}{ }^{\mathrm{t}}$

Difference between the actual loss rate and probe packet loss rate, $\mathrm{D}$ is calculated and compared with a threshold value $\mathrm{T} 1$. If the Value of $\mathrm{D}$ exceeds the threshold $\mathrm{T} 1$ and flow arrival exceeds threshold value $\mathrm{T} 2$, then the flows are marked as VoIP flows. Otherwise it is marked as Normal Data flows.

Load adapter and triggering policy: The normal packets flows are routed through the single best shortest path. The triggering policy invokes the load adapter when the system is in unbalanced state. The load adapter overrides the default routing policy (no dispersion policy) with multipath routing policy as soon as it becomes active. System load balancing can be measured by using different triggering policies (Shi et al., 2005) like Maximum Queue Length Threshold (MQLT), Buffer Occupancy Threshold (BOT) and Periodic Mapping (PM). The proposed approach uses BOT policy to measure the system load balancing. When queue occupancies of the active users exceed a threshold value, load adapter is invoked. Queue occupancy threshold can be set to the $3 / 4$ of the total available buffer space. Load adapter identifies each passing packet whether it belongs to normal flow or VoIP flows and triggering policy checks the state of the system. When the system is in unbalanced state and the packet belongs to VoIP flows, then these flows are rerouted into the congestion free least loaded path. Otherwise the flows are routed normally by using default routing policy.
QoS constraints on MPLS multi path routing: Multipath routing based dispersion involves traffic splitting that consists of dividing each flow of packet stream along two or more disjoint paths such that the sum of available bandwidths of these paths satisfies the bandwidth requirement of each type of flow, depending on the type of traffic class.

The QoS constraint based MPLS Multipath routing finds best shortest paths $(\mathrm{p} 1, \mathrm{p} 2, \mathrm{p} 3) \in \mathrm{P}$, where $\mathrm{p} 1, \mathrm{p} 2$ and $\mathrm{p} 3$ are disjoint loop less paths, that satisfies the QoS specifications. Bandwidth and delay are the two QoS constraints considered in the proposed approach.

The maximum bandwidth and minimum delay required by the end-to-end flow are given as Mbw and Md correspondingly.

Then sum of the bottleneck bandwidths in the disjoint paths should be equal or greater than the bandwidth required by the end-to-end flows to meet the given QoS requirements can be given as Eq. 5:

(ie) For any three paths p1, p2 and p3, $\mathrm{bw}(\mathrm{p} 1)+\mathrm{bw}(\mathrm{p} 2)+\mathrm{bw}(\mathrm{p} 3)>\mathrm{Mbw}$

where, bw(pi) represents bandwidth of the each individual path pi.

Similarly the sum of the delays of the three disjoint paths should be less than minimum required delay, $\mathrm{Md}$ is given by the Eq. 6 :

(ie) For any three paths p1, p2 and p3, $\mathrm{D}(\mathrm{p} 1)+\mathrm{D}(\mathrm{p} 2)+\mathrm{D}(\mathrm{p} 3)<\mathrm{Md}$

where, $\mathrm{D}\left(\mathrm{p}_{\mathrm{i}}\right)$ represents delay of the each individual path $p_{i}$ and $D(p 1)=\sum_{i, j \in p 1} D i j$ and $D(p 2)=\sum_{i, j \in p 2} D i j, D_{i j}$ is the delay along the link $\mathrm{i}$ to $\mathrm{j}$.

These QoS constraint are important for VoIP traffic flows (i.e. with guaranteed levels of Quality of Service) and may be eliminated for best effort traffic.

Packet Dispersion Methodology: The triggering policy invokes the load adapter to override the default routing policy (no dispersion policy) with multipath routing policy when the system is in unbalanced state. The proposed multipath routing policy uses periodic round robin scheduling where the packets are dispersed into multiple label switched path which satisfies the given QoS constraints in a round robin fashion (cyclic manner) over the paths. The deterministic round robin dispersion strategy is a special case of the periodic dispersion. Packet losses at the applications level are caused by many reasons such as network delay and congestion. Therefore in this periodic round robin 
scheduling constant packet loss rate is assumed and losses experienced by the applications are studied. IETF has proposed Noticeable Loss Rate (NLR) and loss constraint distant (Koodli and Ravikanth, 2002) as a metrics for packet loss. Loss constraint distance $(\delta)$ is the threshold value for distance between two losses. NLR is the percentage of lost packets with loss distance smaller than the loss constraint distance. Loss distance is defined as the difference in sequence numbers between two successively loss packets.

Noticeable loss rate (Haim, 2003): for session i with loss constraint $(\delta)$ and for a sequence of k packets, is then given as Eq. 7:

$\mathrm{NLR}_{\mathrm{i}}^{(\delta)}(\mathrm{K})=1 / \mathrm{K} \sum \mathrm{NL}_{\mathrm{i}}^{(\delta)}(\mathrm{S})$

\section{RESULTS AND DISCUSSION}

Simulation setup: The proposed technique is simulated with the network simulator (ns-2) Network simulator. The topology consists of one sender (ingress) and destination (egress) node. These nodes connected to 10 MPLS enabled Label Switched Routers (LSRs). Different link bandwidth and delay are assigned for the 3 paths. Both CBR and VoIP traffic with random exponential loss rate of 0.05 . QoS metrics like received bandwidth, end-to-end delay and packet Loss Rate (NLR) are taken for evaluation. The proposed technique is compared with no dispersion strategy.

Experimental results-simulation load: A low load scenario is considered by varying the load from 250 to 1250 bytes at regular intervals and a high load scenario varies the load from 1500 to 2000 bytes. The QoS parameters such as throughput, end_to_end delay and packet loss rate are measured.

Generally, congestion increased propositionally when the load increased and hence there will be heavy delay. When the load is increased, the proposed adaptive load trigger triggers the packet dispersion strategy so that packets are dispersed into multiple paths which decrease the packet loss and delay. Received throughput is $1.4 \%$ more for multipath dispersion than the no dispersion under low load strategy is shown in Fig. 2.

When the load is increased $1500-2500$ bytes throughput increases $1.3 \%$ in dispersion strategy when compared with no dispersion strategy is shown in Fig. 3. Similarly under low load, the delay is $6 \%$ less in periodic round robin dispersion, when compared to no dispersion is shown in Fig. 4. When the load is increased heavily, delay is reduced by $2 \%$ in multipath dispersion strategy when compared with no dispersion is observed in Fig. 5.

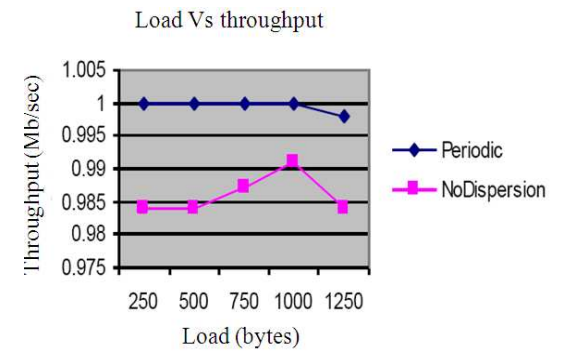

Fig 2: Load Vs throughput (Low Load)

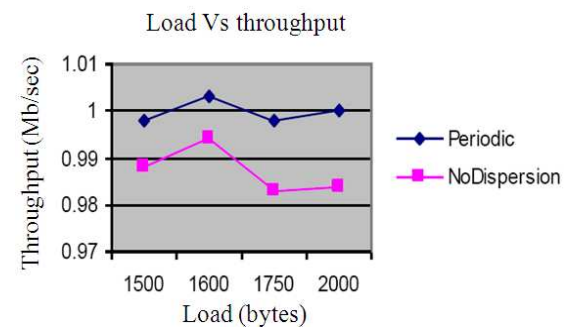

Fig. 3: Load Vs throughput (High Load)

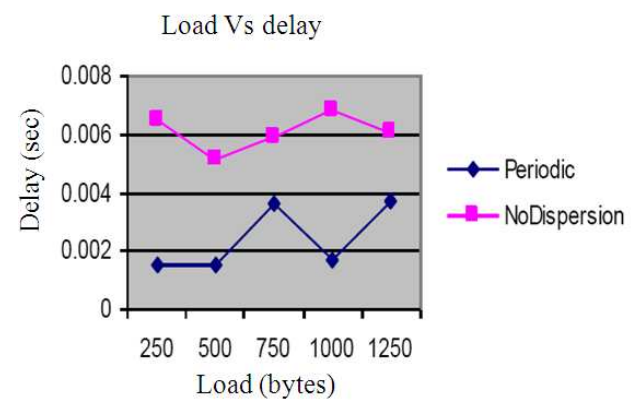

Fig. 4: Load Vs delay (Low Load)

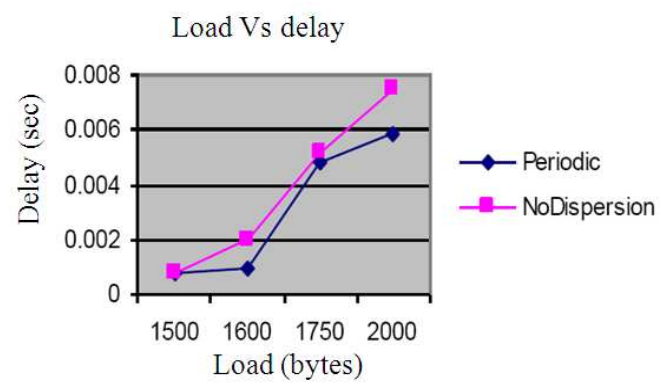

Fig. 5: Load Vs delay (High Load) 


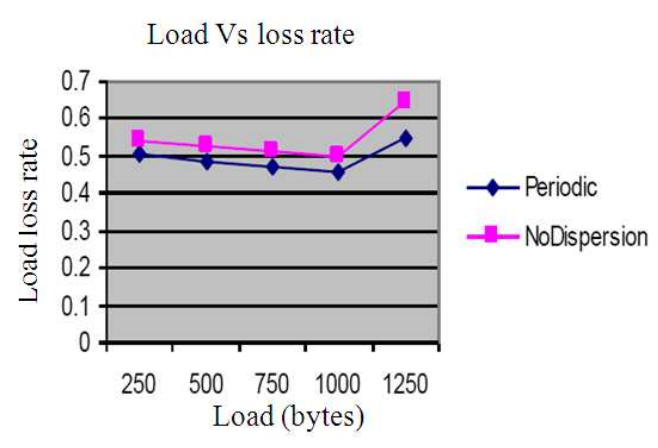

Fig. 6: Load Vs packet loss (Low Load)

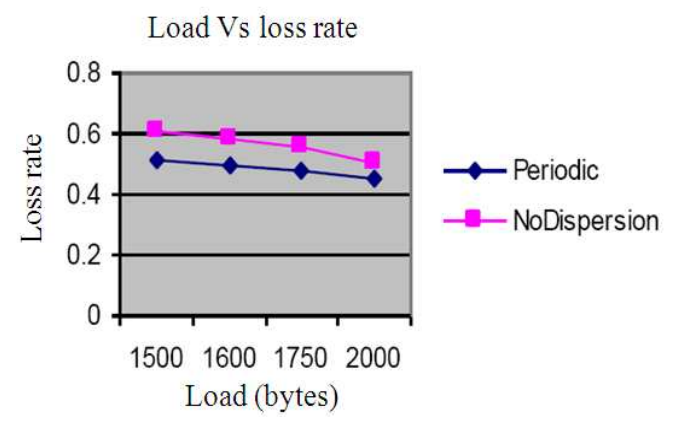

Fig. 7: Load Vs packet loss (High Load)

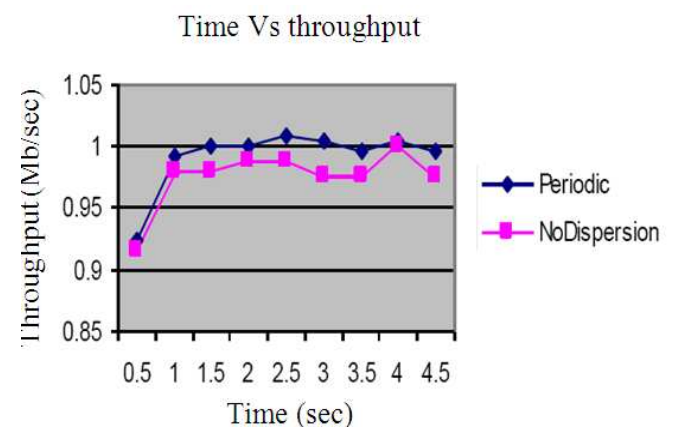

Fig. 8: Time Vs throughput

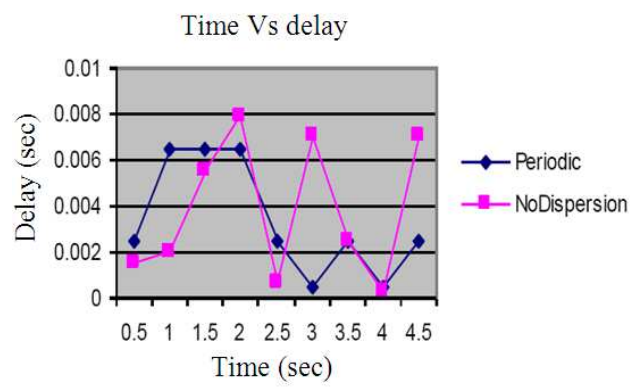

Fig. 9: Time Vs delay

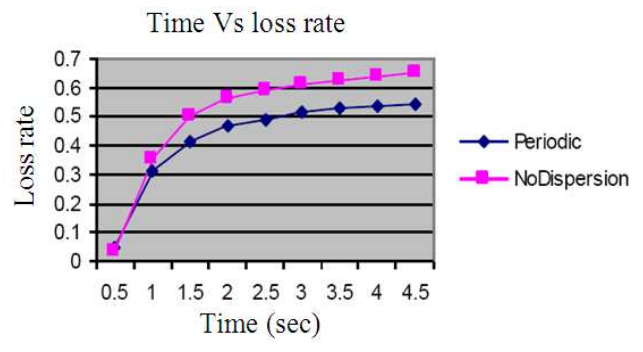

Fig. 10: Time Vs packet loss

It is observed from Fig. 6 that, the packet loss rate is $2.3 \%$ more in the no dispersion strategy and least in the proposed multipath dispersion when the system works under low load. When the load is increased heavily the packet loss rate is reduced by $3.2 \%$ in the periodic round robin dispersion than no dispersion strategy is shown in Fig. 7.

Simulation time: As similar to previous experiment, the throughput, packet loss rate and delay metrics are measured for various time intervals with a fixed load size. $1.6 \%$ of throughput is increased in dispersion strategy than no dispersion strategy is shown in Fig. 8. Similarly $6 \%$ of delay and $2 \%$ of packet loss rate are observed in dispersion strategy when compared with no dispersion strategy is shown in Fig. 9-10.

\section{CONCLUSION}

In this study, Multipath Adaptive Packet Dispersion for Voice Applications is proposed that contains a flow classifier which classifies the flows as VoIP and non VoIP flows. The triggering policy is proposed to check the buffer occupancy value with the buffer occupancy threshold value to evaluate load balancing state in the system. When the load is in unbalanced state, the Load adapter is invoked to disperse the VoIP flows using periodic round robin scheduling along the congestion free, QoS guaranteed multiple paths. It is observed that the proposed system increases the throughput and decreases the packet loss rate and delay by $1.6 \%, 2 \%$ and $6 \%$ respectively when the system works under a fixed load size. By various simulation results, it is shown that the proposed technique attains good throughput with less delay and packet drop, when compared with no dispersion strategy. The superiority of MPLS based Adaptive Packet Dispersion implies that this strategy can improve the VoIP application quality regardless of system load. Due to this improvement dispersion algorithm can be incorporated in the MPLS router to take automatic dispersion decisions based on the current network conditions. As future work, this 
strategy can be extended into interactive streaming applications.

\section{REFERENCES}

Avallone, S., V. Manetti, M. Mariano and S.P. Romano, 2007. A splitting infrastructure for load balancing and security in an MPLS network. Proceedings of the IEEE 3rd International Conference on Testbeds and Research Infrastructure for the Development Network and Communities, May, 21-23, IEEE Xplore Press, Lake Buena Vista, FL., pp: 1-6. DOI: 10.1109/TRIDENTCOM.2007.4444681

Fernandez, E.B., J.C. Pelaez and M.M. Larrondo-Petrie, 2007. Security patterns for voice over IP networks. Proceedings of the International Multi-Conference on Computing in the Global Information Technology, Mar. 4-9, IEEE Xplore Press, Guadeloupe City, pp: 33-33. DOI: 10.1109/ICCGI.2007.57

Guillen, E.P. and D.A. Chacon, 2009. VoIP networks performance analysis with encryption systems. World Acad. Sci. Eng. Technol., 58: 688-695.

Haim, Z., 2003. Packet Dispersion and the Quality of Voice over IP Applications in IP networks. MSc. Thesis, Tel-Aviv University.

Huanga, Y., R. Guerina and P. Gupta, 2006. Supporting excess real-time traffic with active drop queue. IEEE/ACM Trans. Netw., 14: 965-977. DOI: 10.1109/TNET.2006.882902

Kambourakis, G., D. Geneiatakis, T. Dagiuklas, C. Lambrinoudakis and S. Gritzalis, 2006. Towards effective SIP load Balancing. University of the Aegean.
Kandula, S., D. Katabi, S. Sinha and A. Berger, 2007. Dynamic load balancing without packet reordering. ACM SIGCOMM Comput. Commun. Rev., 37: 5162. DOI: $10.1145 / 1232919.1232925$

Koodli, R. and R. Ravikanth, 2002. One-way loss pattern sample metrics. Network Working Group.

Levy, H. and H. Zlatokrilov, 2006. The effect of packet dispersion on voice applications in IP Networks. IEEE/ACM Trans. Netw., 14: 277-288. DOI: 10.1109/TNET.2006.872543

Maheswari, K. and M. Punithavalli, 2009. An improved packet loss recovery in VoIP using combined source and receiver based technique. Int. J. Recent Trends Eng., 2: 120-122.

Molnar, K., J. Hosek, L. Rucka, D. Komosny and M. Vlcek, 2009. Performance evaluation of quality of service assurance in MPLS networks. IJCSNS Int. J. Comput. Sci. Netw. Security, 9: 114-120.

Paganini, F. and E. Mallada, 2009. A unified approach to congestion control and node-based multipath routing. IEEE/ACM Trans. Netw., 17: 1413-1426. DOI: 10.1109/TNET.2008.2011902

Shi, W., M.H. MacGregor and P. Gburzynski, 2005. Load Balancing for parallel forwarding. IEEE/ACM Trans. Netw., 13: 790-801. DOI: 10.1109/TNET.2005.852881

Zlatokrilov, H. and H. Levy, 2004. Packet dispersion and the quality of voice over IP applications in IP networks. Proceeding of the 23rd Annual Joint Conference on the IEEE Computer and Communication Societies, Mar. 7-11, IEEE Xplore Press, $\quad$ pp: 1170-1180. DOI: 10.1109/INFCOM.2004.1357003 